University of Nebraska - Lincoln

DigitalCommons@University of Nebraska - Lincoln

Timothy J. Gay Publications

Research Papers in Physics and Astronomy

2010

Electron-spin-reversal phenomenon in optically pumped rubidium

\author{
E. B. Norrgard \\ University of Nebraska - Lincoln \\ D. Tupa \\ Los Alamos National Laboratory \\ J. M. Dreiling \\ University of Nebraska - Lincoln \\ Timothy J. Gay \\ University of Nebraska - Lincoln, tgay1@unl.edu
}

Follow this and additional works at: https://digitalcommons.unl.edu/physicsgay

Part of the Physics Commons

Norrgard, E. B.; Tupa, D.; Dreiling, J. M.; and Gay, Timothy J., "Electron-spin-reversal phenomenon in optically pumped rubidium" (2010). Timothy J. Gay Publications. 66.

https://digitalcommons.unl.edu/physicsgay/66

This Article is brought to you for free and open access by the Research Papers in Physics and Astronomy at DigitalCommons@University of Nebraska - Lincoln. It has been accepted for inclusion in Timothy J. Gay Publications by an authorized administrator of DigitalCommons@University of Nebraska - Lincoln. 


\title{
Electron-spin-reversal phenomenon in optically pumped rubidium
}

\author{
E. B. Norrgard, ${ }^{1}$ D. Tupa, ${ }^{2}$ J. M. Dreiling, ${ }^{1}$ and T. J. Gay ${ }^{1}$ \\ ${ }^{1}$ Behlen Laboratory of Physics, University of Nebraska, Lincoln, Nebraska 68588-0111, USA \\ ${ }^{2}$ Los Alamos National Laboratory, Los Alamos, New Mexico 87545, USA
}

(Received 1 May 2010; published 10 September 2010)

\begin{abstract}
We have studied the optical pumping of mixtures of $\mathrm{Rb}$ vapor and $\mathrm{N}_{2}$ buffer gas by laser light tuned to the $D_{1}$ transition having a spectral width of $\sim 500 \mathrm{MHz}$. The Rb densities are of the order of $10^{13} \mathrm{~cm}^{-3}$, while the buffer-gas pressures range from 0.1 to 10 torr. As the frequency of the right-hand circularly polarized laser is varied across the $D_{1}$ absorption profile, the electron spin polarization of the $\mathrm{Rb}$ is found to take on negative values for small negative values of pump detuning from the absorption profile center. This occurs for $\mathrm{N}_{2}$ pressures below $\sim 1$ torr; at 10 torr the electron spins consistently point in the same direction as the angular momentum of the pump light. The spin-reversal effect can be understood in terms of populations of the $F=2\left({ }^{85} \mathrm{Rb}\right)$ and $F=1\left({ }^{87} \mathrm{Rb}\right)$ states caused by small unpolarized fractions in the pump beam and its elimination in terms of pressure broadening caused by the $\mathrm{N}_{2}$ buffer gas. We speculate that this effect could be used for fast $\mathrm{Rb}$ spin modulation.
\end{abstract}

DOI: 10.1103/PhysRevA.82.033408

PACS number(s): $32.80 . X x$

\section{INTRODUCTION}

We report here an interesting phenomenon in optical pumping experiments: the production of overall electron spin polarization in an alkali-metal vapor oriented antiparallel to the spin of the pump light. Optical pumping of alkali-metal vapors is an important tool in atomic physics. It is used in a broad range of experiments such as the storage of light [1], atomic clocks [2], the production of polarized ions [3] and neutrons [4], and the generation of polarized noble gases through spin-exchange optical pumping [5]. Although the fundamental techniques of these experiments are similar, they operate under very different conditions. Storage of light in a warm alkali-metal vapor is effected with little or no buffer gas and with spectrally narrow, low-power lasers, while systems that use spin-exchange optical pumping to generate polarized noble gases tend to use high buffer-gas pressures ( $\sim 1000$ torr) and broad, high-power lasers. The work reported here deals with a third regime, using somewhat broad, high-power lasers with low pressures ( $\sim 1$ torr) of buffer gas [6,7]. These conditions are optimal to generate a beam of electrons polarized through spin exchange with a spin-polarized optically pumped alkali-metal target [7]. The necessity of passing the electrons through the alkali-metal $(\mathrm{Rb})$ vapor requires that the buffer-gas pressures be modest to maintain an appreciable electron current. We hope to use this technology to produce a "turnkey" polarized electron source.

In order to better understand our source performance, we undertook a systematic study to characterize the rubidium polarization under these conditions.

\section{EXPERIMENT}

Our experiments are conducted in a test cell with no electron beam present. We use a pumping laser with intermediate spectral width: $\sim 500 \mathrm{MHz}$. This choice allows us to selectively pump pairs of low-field $D_{1}$ transitions with a common hyperfine ground level and an unresolved excited hyperfine doublet while also efficiently pumping their Doppler- and collisionally-broadened absorption profiles, which are also $\sim 500 \mathrm{MHz}$ wide in our measurements. The optical layout of our experiment is shown schematically in Fig. 1. A 10-W beam from a Coherent Verdi laser is used to pump a Spectra Physics 3900 Ti:sapphire laser. The output of this laser is $\sim 1 \mathrm{~mm}$ in diameter with a typical power of $800 \mathrm{~mW}$. The beam passes through a clean-up linear polarizer and quarter-wave plate before entering a test cell $\sim 5 \mathrm{~cm}$ long containing $\mathrm{Rb}$ vapor with a natural isotopic abundance. The Rb density, $N_{\mathrm{Rb}}$, is $\sim 10^{13} \mathrm{~cm}^{-3}$ in this cell, comparable to the density used for our optically pumped $\mathrm{Rb}$ electron spin filter [7]. The cell also includes $\mathrm{N}_{2}$ buffer gas at a pressure of $0.1,1.0$, or 10 torr. A variable longitudinal magnetic field of up to $0.025 \mathrm{~T}$ is applied to the cell.

An absorption spectrum of the probe beam in the roomtemperature $\mathrm{Rb}$ reference cell $\left(N_{\mathrm{Rb}} \sim 10^{10} \mathrm{~cm}^{-3}\right)$ that contains no buffer gas is shown in Fig. 2(a). This is used to provide a fiducial frequency marker for the probe detuning values in the pumping cell. In the reference cell absorption profile, the hyperfine structure of the Rb is apparent, with the widths of the individual absorption dips being determined primarily by Doppler broadening, with contributions from probe laser power broadening. Because the eight individual $D_{1}$ hyperfine transitions overlap significantly due to broadening, Fig. 2(b) is provided to indicate the position of the line center of each transition. The pumping frequency is determined within $0.5 \mathrm{GHz}$ using a Burleigh WA-1000 wave meter. In the test cell, the pressure broadening due to $\mathrm{N}_{2}$ is $18 \mathrm{MHz} /$ torr [8]. Doppler widths in the test cell are $\sim 500 \mathrm{MHz}$ at all the temperatures we consider.

We use methods described elsewhere $[6,9]$ to determine $N_{\mathrm{Rb}}$ and the $\mathrm{Rb}$ polarization, $P_{\mathrm{Rb}}$, with the Faraday rotation angles $\Delta \phi_{N}$ and $\Delta \phi_{P}$, respectively, of the probe beam with an imposed magnetic field of strength $B$. The probe beam detunings, $\delta$, are kept sufficiently large $(>15 \mathrm{GHz})$ and its intensity kept low enough (typically $20 \mu \mathrm{W}$ ) that it does not alter the $\mathrm{Rb}$ Zeeman sublevel populations caused by the pumping process. Here $P_{\mathrm{Rb}}$ corresponds to the orientation of the $\mathrm{Rb} 5^{2} S_{1 / 2}$ fine-structure ground state:

$$
P_{\mathrm{Rb}}=\frac{n_{\uparrow}-n_{\downarrow}}{n_{\uparrow}+n_{\downarrow}},
$$




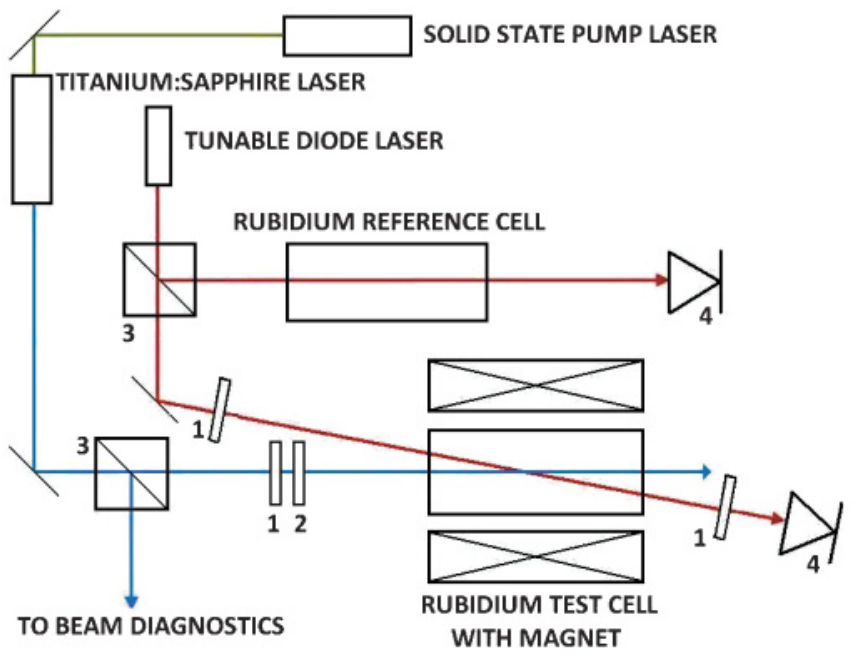

FIG. 1. (Color online) Apparatus schematic: (1) linear polarizer, (2) quarter-wave plate, (3) beam sampler, (4) photodiode.

where $n_{\uparrow}$ is the number of atoms with $m_{J}=+1 / 2$ and $n_{\downarrow}$ is the number of atoms with $m_{J}=-1 / 2$. This is in turn equivalent to the $\mathrm{Rb}$ electron polarization. The uncertainties in our measurements of $N_{\mathrm{Rb}}$ and $P_{\mathrm{Rb}}$ are due to uncertainties in $\delta, \Delta \phi_{N}, \Delta \phi_{P}, \vec{B}$, and the relative angle between the probe and pump beams. In our measurements, the former two uncertainties dominate. Due to the loss of a test cell during the experiment, the 10-torr data we present were taken at about half the number density as the other two data sets and have noticeably higher uncertainty.

\section{RESULTS}

We measured $P_{\mathrm{Rb}}$ as a function of pump detuning for three buffer-gas pressures: $0.1,1.0$, and 10 torr. The averaged $\mathrm{Rb}$ polarization as a function of detuning is shown in Fig. 2(c). All three curves display positive polarization for positive detunings and larger negative detunings. The magnitude of $P_{\mathrm{Rb}}$ increases with increasing $\mathrm{N}_{2}$ pressure, because the buffer gas inhibits diffusion of $\mathrm{Rb}$ to the cell walls, lowering the spin relaxation rate. For 10-torr $\mathrm{N}_{2}$ pressure, $P_{\mathrm{Rb}}$ is positive at all pumping frequencies. It remains appreciable even for the largest pump laser detunings, a result of the combined effects of pressure and power broadening. Residual hyperfine structure is present, but the excursion of $P_{\mathrm{Rb}}$ below its broad maximum is relatively small. As the $\mathrm{N}_{2}$ pressure is decreased, a pronounced polarization dip appears, to the extent that the electron polarization actually flips sign for small negative detunings. This phenomenon has, to our knowledge, not been reported previously in optical pumping experiments. It corresponds to the counterintuitive result that for certain frequencies, electrons in optically pumped $s$ states "spin the wrong way," that is, antiparallel to the angular momentum of the pump beam.

\section{QUALITATIVE DISCUSSION}

The observed spin reversal arises from the underlying hyperfine structure of the optically pumped $\mathrm{Rb}$ vapor. Consider the Zeeman structure of ${ }^{85} \mathrm{Rb}$ (Fig. 3). In the ground-state hy-

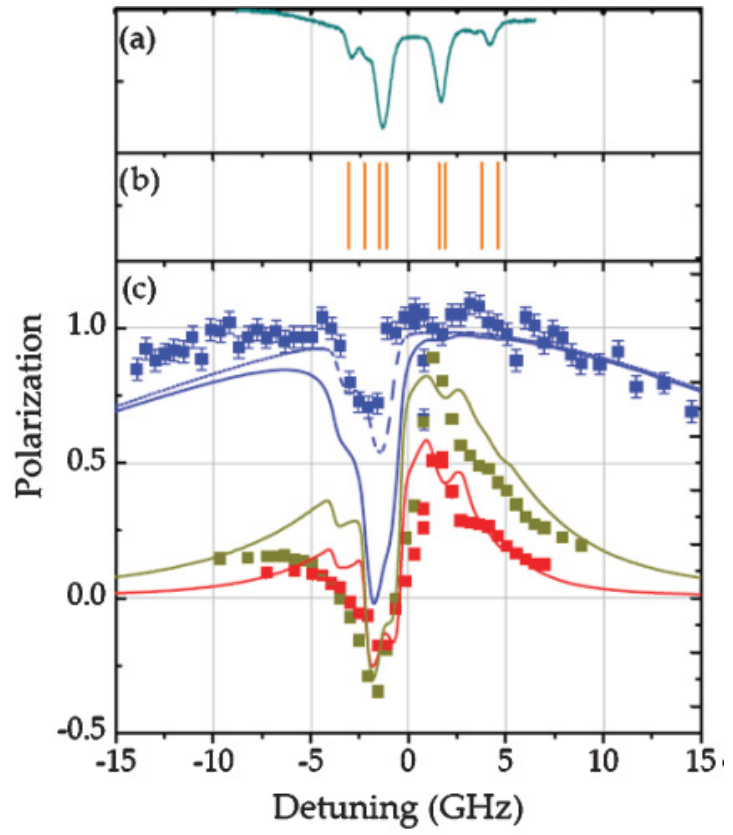

FIG. 2. (Color) (a) Absorption scan of the probe in the $\mathrm{Rb}$ reference cell. (b) The positions of the hyperfine ground $(g)$ to excited (e) level transitions of $\mathrm{Rb}$; from left to right: ${ }^{87} \mathrm{Rb} F_{g}=2 \rightarrow F_{e}=1$, ${ }^{87} \mathrm{Rb} 2 \rightarrow 2,{ }^{85} \mathrm{Rb} 3 \rightarrow 2,{ }^{85} \mathrm{Rb} 3 \rightarrow 3,{ }^{85} \mathrm{Rb} 2 \rightarrow 2,{ }^{85} \mathrm{Rb} 2 \rightarrow 3$, ${ }^{87} \mathrm{Rb} 1 \rightarrow 1,{ }^{87} \mathrm{Rb} 1 \rightarrow 2$. (c) Measured (data points) and calculated (curves) polarization of a natural-abundance $\mathrm{Rb}$ vapor as a function of the pump laser frequency. Red data: 0.1 torr $\mathrm{N}_{2}, 8.4 \times 10^{12} \mathrm{~cm}^{-3}$ $\mathrm{Rb}$ density; red curve: 0.1 torr $\mathrm{N}_{2}, 99.5 \% \sigma^{+}$light polarization. Green data: 1.0 torr $\mathrm{N}_{2}, 8.8 \times 10^{12} \mathrm{~cm}^{-3} \mathrm{Rb}$; green curve: 1.0 torr $\mathrm{N}_{2}, 99.5 \%$ light polarization. Blue data: 10 torr $\mathrm{N}_{2}, 4.3 \times 10^{12} \mathrm{~cm}^{-3} \mathrm{Rb}$; blue solid curve: 10 torr $\mathrm{N}_{2}, 99.5 \%$ light polarization; blue dashed curve: 10 torr $\mathrm{N}_{2}, 99.95 \%$ light polarization.

perfine level $\left({ }^{2} S_{1 / 2}, F=3\right)$ for which $F=I+J$, the electron spin polarization is proportional to the total angular momentum Zeeman quantum number $m_{F}$; in the lower hyperfine level $\left({ }^{2} S_{1 / 2}, F=2\right)$, where $F=I-J$, electron spin polarization is proportional to $-m_{F}$. The process of optical pumping with

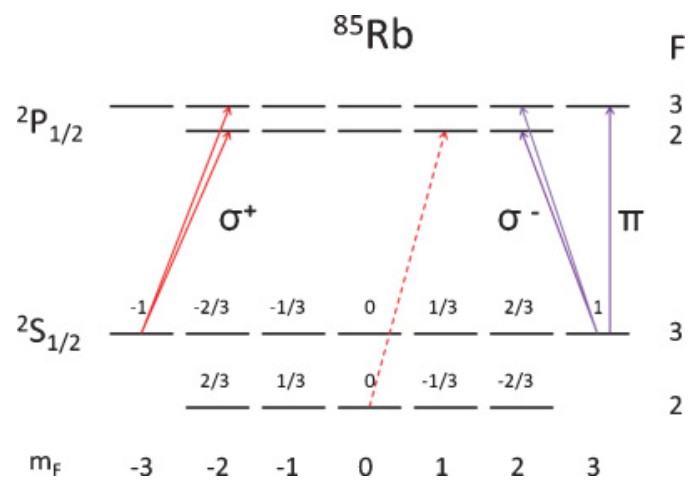

FIG. 3. (Color online) Zeeman level diagram of ${ }^{85} \mathrm{Rb}$. Electron spin polarizations for ground $m_{F}$ states are indicated above the sublevel. With the pump beam along the cell axis, the indicated $\pi$ transition can result only from poor pump-laser collimation or misalignment. The $\sigma^{-}$transitions result from imperfect $\sigma^{+}$polarization. The dashed arrow indicates repumping from the hyperfine ground state caused by pressure broadening. 
right-hand circularly polarized $\left(\sigma^{+}\right)$light tends to enhance the population of states with the highest values of $m_{F}$. Thus, if one could selectively populate the ${ }^{2} S_{1 / 2}, F=2$ level with a positive expectation value of $m_{F}$, it would have negative electron polarization [10].

With some important but subtle issues to be discussed in what follows, this idea explains qualitatively the structure of the $P_{\mathrm{Rb}}$ vs detuning curves taken with low buffer-gas pressure. At small positive detunings from the absorption line center, the pump laser is tuned to the ${ }^{85} \mathrm{Rb} F=2 \rightarrow F=(2,3)$ transitions, and the effect of $\sigma^{+}$light absorption followed by emission is to populate exclusively the $F=3$ ground state and increase $m_{F}$, producing a positive polarization. However, at small negative detunings, the laser is in the vicinity of the ${ }^{85} \mathrm{Rb} F=3 \rightarrow F=(2,3)$ transitions; $m_{F}$ increases though absorption and emission cycles while population transfers to the $F=2$ level. This produces negative electron polarization.

Achieving negative polarization by pumping the $F=3 \rightarrow F=(2,3)$ transition, however, ultimately requires a $\pi$ or $\sigma^{-}$component of the pump laser polarization that can drive vertical or left-going absorptions [11]. In the case of pure $\sigma^{+}$light driving only $F=3 \rightarrow F=(2,3)$ transitions, two problems occur. The $F=3, m_{F}=+3$ dark state becomes sufficiently populated to drive the overall electron polarization positive. Moreover, once decay into the $F=2$ state has occurred, there is no longer a pumping mechanism to shuttle its $m_{F}$ population to the right. For the example of negligible spin relaxation rate, we calculate that $P_{\mathrm{Rb}}$ is +0.09 with pure $\sigma^{+}$pump light. However, with a very small component of $\pi$ or $\sigma^{-}$light in the pump beam, $P_{\mathrm{Rb}}$, is $\sim-0.17$ (depending weakly on the component of the pump light that is $\left.\operatorname{not} \sigma^{+}\right)$.

There are four time scales of interest in this system arising from optical pumping, dark-state depletion, spin relaxation, and repumping. The optical pumping rate is determined by the optical absorption cross section and the pump-laser intensity. It is much greater than all the other rates, and, like the dark-state depletion rate and the repumping rate, it depends on the pump-laser intensity but has a much higher constant of proportionality. A typical value for the optical pumping rate under our conditions is $4 \times 10^{9} \mathrm{~s}^{-1}$. The dark-state depletion rate is that at which the $F=3, m_{F}=+3$ state is emptied and depends on the intensity of non- $\sigma^{+}$polarization. As an example, our calculations (to be discussed below) for $99.5 \%$ pump-light polarization [Fig. 2(c)] correspond to a dark-state depletion rate $\sim 0.005$ times the optical pumping rate, or $2 \times 10^{7} \mathrm{~s}^{-1}$. The spin relaxation and repumping rates depend on the buffer-gas pressure. Because our laser beam diameter is much less than the diameter of the test cell and we have low buffer-gas pressures, the spin relaxation rate is roughly the inverse of the time for an atom to cross the laser beam. At a pressure of 0.1 torr, the collisional mean free path of an atom is greater than the laser beam diameter, and at a pressure of 10 torr an atom undergoes many collisions before it crosses the beam. Thus, the system moves from the molecular-flow to the diffusion regime as the pressure is increased, and the relaxation rate must be determined accordingly. We calculate this rate to be $3 \times 10^{5} \mathrm{~s}^{-1}, 2 \times 10^{5} \mathrm{~s}^{-1}$, and $4 \times 10^{4} \mathrm{~s}^{-1}$ at buffer-gas pressures of $0.1,1.0$, and 10 torr, respectively. We call the rate of pumping from the $F=2$ state the "repumping" rate. It is derived from an integral of the overlap of the pump laser's spectral profile with the pressure-broadened Lorentzian wings of the $F=2 \rightarrow F=(2,3)$ transitions. For a pump laser tuned to the $F=3 \rightarrow F=(2,3)$ frequency, where we see the largest polarization reversal, the repumping rate increases with pressure and has typical values of $1 \times 10^{6} \mathrm{~s}^{-1}, 4 \times 10^{6} \mathrm{~s}^{-1}$, and $3 \times 10^{7} \mathrm{~s}^{-1}$ for $\mathrm{N}_{2}$ pressures of $0.1,1.0$, and 10 torr, respectively.

The spin-reversal phenomenon is seen at intensities of $\pi$ or $\sigma^{-}$light for which the dark-state depletion rate is greater than the repumping rate. With no buffer gas present to cause collisional broadening, the repumping rate is small, and a very small fraction of improper polarization-on the order of $10^{-4}$ - can produce the spin-reversal effect. Indeed, the extinction ratio of a standard polarizing beam splitter shows it to be inadequate to prevent the spin reversal. Another possible cause of imperfect $\sigma^{+}$pump beam polarization would be misalignment with the magnetic field or imperfect collimation; a mutual misalignment or a laser miscollimation of less than $1^{\circ}$ is sufficient to cause a spin reversal.

The sign of $P_{\mathrm{Rb}}$ depends on the sign of the difference between the repumping and dark-state depletion rates. Its magnitude depends on the absolute value of this difference compared to the spin relaxation rate. When $P_{\mathrm{Rb}}$ is positive, its value tends to +1.0 . It does not reach -1.0 under spin-reversal conditions because the highest $m_{F}$ state in the $F=2$ ground level has an electron polarization of only $-2 / 3$. Moreover, the pumping process for this level does not shuttle the atoms to higher $m_{F}$ efficiently because the laser is tuned to the $F=3 \rightarrow F=(2,3)$ frequency.

To explain the disappearance of the spin reversal as the $\mathrm{N}_{2}$ pressure reaches 10 torr, consider the case where the pump laser is tuned to the $F=3 \rightarrow F=(2,3)$ frequency with a negative detuning of $\sim 1.5 \mathrm{GHz}$, the optimal value for producing $P_{\mathrm{Rb}}<0$. As the buffer-gas pressure is increased, collisional broadening makes the pumping more effective in the wings of the $F=2 \rightarrow F=(2,3)$ transitions, increasing the repumping rate. Atoms more easily leave the $F=2$ level, with the effect of first diminishing and then reversing the sign of $P_{\mathrm{Rb}}$ at this frequency. The transition from $P_{\mathrm{Rb}}<0$ to $P_{\mathrm{Rb}}>0$ occurs when the buffer-gas pressure is just high enough to produce a repumping rate exceeding the dark-state depletion rate.

\section{MODEL CALCULATIONS}

We have modeled these effects with rate equations for the individual $F, m_{F}$, and ground- and excited-state sublevels for ${ }^{85} \mathrm{Rb}$ and ${ }^{87} \mathrm{Rb}$. We make a number of assumptions to simplify the calculations. The laser beam is taken to be spatially flat in both radius and distance along the cell axis. The spectral profile of our pump laser has three laser cavity modes equally spaced $\sim 200 \mathrm{MHz}$ apart; we approximate this with a spectrally flat beam of width $550 \mathrm{MHz}$, having an adjustable superposition of $\sigma^{+}$and $\sigma^{-}$light. It is essential to describe the optical absorption with a Voigt profile convolution of the Doppler-broadened Gaussian line shape with the natural width [12] and collisional Lorentzian line shape. We neglect individual velocity subgroups and consider each atom to have this idealized line shape. The laser-induced pump, dark-state 
depletion, and repump rates are obtained by integrating the overlap of the laser spectral profile with the absorption line shape for the relevant transitions. The spin relaxation rate is the beam-crossing rate for atoms in vacuum joined smoothly to the beam-crossing rate for atoms diffusing in the presence of $\mathrm{N}_{2}$ gas to account for the transition from the molecular flow to diffusion regimes. The intensity, diameter, and $\sigma^{-}$-polarized fraction of the pumping light are the only free parameters in the calculations. Since spin-exchange collisions are not important in this regime, our results are independent of $N_{\mathrm{Rb}}$. Thus, we solve the rate equations for ${ }^{87} \mathrm{Rb}$ and ${ }^{85} \mathrm{Rb}$ independently and combine their results in a weighted average.

The predictions of the model are depicted as the solid-line curves in Fig. 2(c) with the fit parameters of laser power $=$ $100 \mathrm{~mW}$, the radius $=0.5 \mathrm{~mm}$, and the fraction of $\sigma^{-}$polarized light $=0.005$. The model gives reasonable agreement in the overall shape of the data, depicting the appearance of the negative polarization at 0.1 torr buffer pressure, the deepening of the dip at the intermediate pressure, and the ultimate decrease of the dip at 10 torr. It also gives good agreement with the heights of the positive excursions and the shapes of the wings. The dashed-line curve shows the results for 10 torr with the fraction of $\sigma^{-}$polarized light $=0.0005$. As the data sets were taken on different days, the wave plate may have been set slightly differently for the 10-torr data set or experienced a small rotation around the vertical axis, which would yield different polarizations at the respective optimal settings.

As an aside, we note that a repumping mechanism could, in principle, result from spin-exchange collisions between $\mathrm{Rb}$ atoms [5]. This method of redistributing atoms between the ground state $F$ levels is not so relevant under conditions for a polarized electron source because a quench gas cannot be used in sufficient quantity to mitigate the radiation trapping. Thus $N_{\mathrm{Rb}}$ for which spin-exchange collisions become a factor is above that for which radiation trapping limits the polarization $[13,14]$.

\section{CONCLUSIONS}

We have presented data sets for $P_{\mathrm{Rb}}$ as a function of laser detuning and $\mathrm{N}_{2}$ buffer-gas pressure. Under certain conditions, the data display a reversal of the electron spin polarization for some laser frequencies, even if the helicity of the circularly polarized laser beam remains unchanged. We have developed a model showing that this behavior is due to a combination of imperfect laser polarization, Rb hyperfine structure, and a repumping effect caused by buffer-gas pressure broadening. Our data and model elucidate the transition from negligible buffer-gas pressure, where a repumping laser is used to empty the undesired ground-state hyperfine level, to buffer-gas pressures where the hyperfine components of the ground state are sufficiently unresolved that a single laser is adequate to obtain high electron spin polarization. Furthermore, we demonstrate that a spin reversal can be effected by optically pumping with a single laser and merely shifting its frequency. This offers the prospect of a quick, inexpensive, and convenient way to modulate the overall spin of a $\mathrm{Rb}$ sample. Our model predicts, for example, that it is possible to alternate between polarizations of $+40 \%$ and $-40 \%$ at frequencies well in excess of $1 \mathrm{kHz}$. These results are significant for applications employing low buffer-gas pressures, such as polarized electron sources or polarized $\mathrm{H}$ or $\mathrm{D}$ gas targets [15-18].

\section{ACKNOWLEDGMENTS}

The authors acknowledge very helpful discussions with Mike Romalis and Herman Batelaan. This work was supported by NSF Grant No. PHY-0855629 and is unclassified: Los Alamos Unlimited Release LA-UR 10-02789.
[1] D. F. Phillips, A. Fleischhauer, A. Mair, R. L. Walsworth, and M. Lukin, Phys. Rev. Lett. 86, 783 (2001).

[2] Y.-Y. Jau, A. B. Post, N. N. Kuzma, A. M. Braun, M. V. Romalis, and W. Happer, Phys. Rev. Lett. 92, 110801 (2004).

[3] L. W. Anderson, Nucl. Instrum. Methods 167, 363 (1979).

[4] B. Chann, E. Babcock, L. W. Anderson, T. G. Walker, W. C. Chen, T. B. Smith, A. K. Thompson, and T. R. Gentile, J. Appl. Phys. 94, 6908 (2003).

[5] T. G. Walker and W. Happer, Rev. Mod. Phys. 69, 629 (1997).

[6] M. A. Rosenberry, J. P. Reyes, D. Tupa, and T. J. Gay, Phys. Rev. A 75, 023401 (2007).

[7] H. Batelaan, A. S. Green, B. A. Hitt, and T. J. Gay, Phys. Rev. Lett. 82, 4216 (1999).

[8] M. E. Wagshul and T. E. Chupp, Phys. Rev. A 49, 3854 (1994).

[9] Z. Wu, M. Kitano, W. Happer, M. Hou, and J. Daniels, Appl. Opt. 25, 4483 (1986).
[10] In earlier experiments that limited the observation to a subset of atoms in the lower hyperfine level, an electron spin antiparallel to the spin of the pump light was reported. W. Happer and W. A. Winjngaarden, Hyperfine Interact. 38, 435 (1987); F. A. Franz, Phys. Rev. 141, 105 (1966).

[11] Mike Romalis (Princeton University) made us aware of the importance of a non- $\sigma^{+}$fraction in the pump beam for the production of negative polarization.

[12] D. A. Steck, Rubidium-87 D Line Data (2003) [http://steck.us/ alkalidata/].

[13] D. Tupa, L. W. Anderson, D. L. Huber, and J. E. Lawler, Phys. Rev. A 33, 1045 (1986).

[14] D. Tupa and L. W. Anderson, Phys. Rev. A 36, 2142 (1987).

[15] J. Seely et al., Phys. Rev. A 73, 062714 (2006).

[16] A. Airapetian et al., Nucl. Instrum. Methods 540, 68 (2005).

[17] R. V. Cadman et al., Phys. Rev. Lett. 86, 967 (2001).

[18] Z.-L. Zhou et al., Nucl. Instrum. Methods 378, 40 (1996). 